\title{
Detection of Interstitial Atoms by Hollow-Cone Illumination HAADF-STEM
}

\author{
K. Saitoh and N. Tanaka
}

EcoTopia Science Institute, Nagoya University, Furo-cho, Chikusa-ku, Nagoya, 464-8603

When a fast electron propagates through a crystalline specimen along a zone-axis, the electron selectively passes through a certain atomic column site. This channeling behavior of the propagating electron is approximately the same for a fine probe of scanning transmission electron microscopy (STEM). The STEM probe is a conical illumination beam with a convergence semi-angle of a few tens of mrad. In a zone-axis incidence, the beam passes predominantly through an atomic column site [1,2], because the probe has a large portion of plane wave components, which are nearly along the zone axis. The atomic column channeling of the STEM probe plays an important role for Z-contrast imaging by using high-angle scattered electrons, or HAADF-STEM. That is, HAADF-STEM is sensitive only to structures of well-channeled atomic columns, but is insensitive to those of the weakly channeled sites or impurities located at interstitial sites.

In the present study, we investigate a possibility for the enhancement of detecting the interstitial atoms and light elements by using a hollow-cone illumination, which can excite "interstitial channeling". We performed Bloch wave calculations for revealing the propagation of the incident electron in the hollow-cone illumination mode.

Figure 1 shows the atomic arrangement of silicon projected onto the $<110>$ direction. A normal conical beam (convergence semi-angle: 20mrad) and a hollow-cone beam (inner semi-angle: $20 \mathrm{mrad}$, outer semi-angle: $26 \mathrm{mrad}$ ) with an accelerating voltage of $200 \mathrm{kV}$ are situated at the position indicated by the cross as incident beams. The positions of the silicon columns are overlaid on the map of the $5 \mathrm{~nm}$ depth. Figure 2 shows intensity distributions of the propagating electron for specimen depths from $5 \mathrm{~nm}$ to $30 \mathrm{~nm}$ with the normal cone beam (upper) and the hollow-cone beam (lower). The center of each map corresponds to the probe position. In the case of the normal cone beam, the intensity is localized around the probe position at $5 \mathrm{~nm}$ depth and starts scattering at 10 $\mathrm{nm}$ depth. At $15 \mathrm{~nm}$ depth, the intensity has peaks at the neighboring silicon columns, indicating the formation of the atomic column channeling. In the hollow-cone beam case, the intensity maps show strong peaks at the probe position and diffuse intensities around the peaks for all the depths. This indicates that the interstitial channeling is excited by the hollow-cone illumination. Intensity map for other probe positions and other orientations were also investigated. Interstitial channeling was also confirmed in the other crystalline orientations. Experiments with the hollow-cone illumination is in progress.

One of the authors (K.S.) is thankful to Dr. K. Tsuda for providing source codes of his Bloch wave calculation program [3]. 
References

[1] P. D. Nellist and S. J. Pennycook, Ultramicroscopy 78 (1999) 111.

[2] C. Dwyer, J. Etheridge: Ultramicroscopy 96 (2003) 343.

[3] K. Tsuda and M. Tanaka: Acta Cryst. A55 (1999) 939.

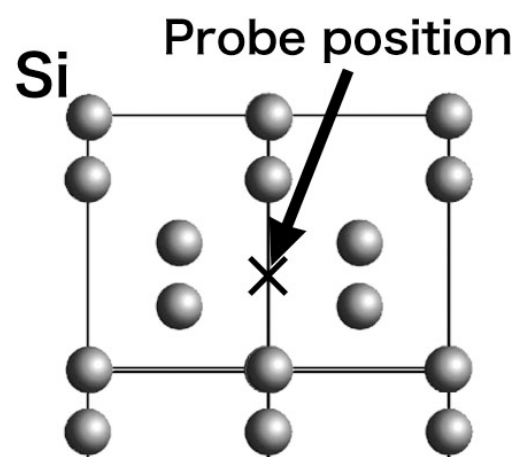

Figure 1: Atomic arrangement of silicon projected in the $<110>$ direction. Probe is situated at the position indicated by the cross.
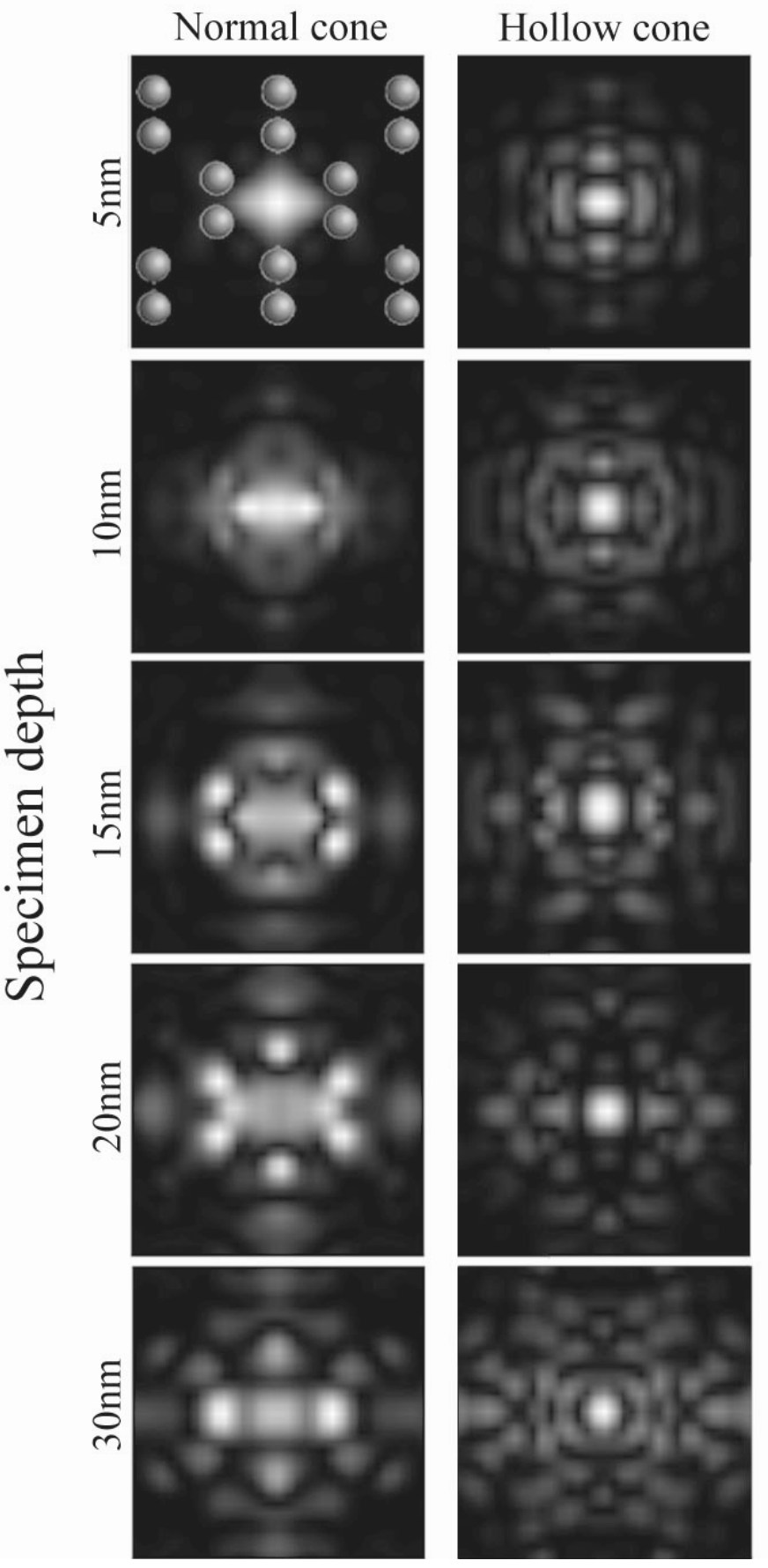

Figure 2: Intensity distributions of the propagating electrons with a normal cone beam (left) and with a hollow-cone beam (right). 